\title{
Functional Characterization of a Novel Amidase Involved in Biotransformation of Triclocarban and its Dehalogenated Congeners in Ochrobactrum sp. TCC-2
}

\author{
Hui Yun, ${ }^{\dagger, \ddagger}$ Bin Liang, ${ }^{* \dagger \oplus}$ Jiguo Qiu, ${ }^{\S}$ Long Zhang, ${ }^{\S}$ Youkang Zhao," Jiandong Jiang, ${ }^{\S}$ \\ and Aijie Wang*, ${ }^{\dagger}, \|$
}
${ }^{\dagger}$ Key Laboratory of Environmental Biotechnology, Research Center for Eco-Environmental Sciences, Chinese Academy of Sciences, Beijing, 100085, China
${ }^{\ddagger}$ University of Chinese Academy of Sciences, Beijing, China
${ }^{\S}$ Key Lab of Microbiological Engineering of Agricultural Environment, Ministry of Agriculture, College of Life Sciences, Nanjing Agricultural University, 210095, Nanjing, China
"State Key Laboratory of Urban Water Resource and Environment, Harbin Institute of Technology, Harbin, 150090, China

Supporting Information

\begin{abstract}
Haloaromatic antimicrobial triclocarban (3,4,4'-trichlorocarbanilide, TCC) is a refractory contaminant which is frequently detected in various aquatic and sediment environments globally. However, few TCC-degrading communities or pure cultures have been documented, and functional enzymes involved in TCC biodegradation hitherto have not yet been characterized. In this study, a bacterial strain, Ochrobactrum sp. TCC-2, capable of degrading TCC under both aerobic and anaerobic conditions was isolated from a sediment sample. A novel amidase gene $(t c c A)$, responsible for the hydrolysis of the two amide bonds of TCC and its dehalogenated congeners 4,4'-dichlorocarbanilide (DCC) and carbanilide (NCC) to more biodegradable chloroaniline or aniline products, was cloned and characterized. TccA shares low amino acid sequence identity

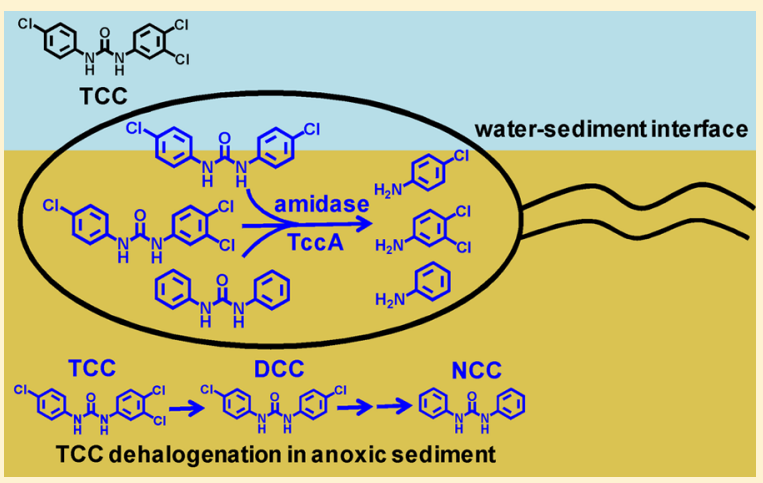
(27 to $38 \%$ ) with other biochemically characterized amidases and contains the conserved catalytic triad (Ser-Ser-Lys) of the amidase signature enzyme family. TccA was stable over a $\mathrm{pH}$ range of 5.0 to 10.0 and at temperatures lower than $60^{\circ} \mathrm{C}$, and it was constitutively expressed in strain TCC-2. In contrast to the halogenated TCC and DCC, the nonchlorinated NCC was the preferred substrate for TccA. TccA also had hydrolysis activity to a broad spectrum of amide bonds in herbicides, insecticides, and chemical intermediates. The constitutive expression and broad substrate spectrum of TccA suggested strain TCC-2 may be potentially useful for bioremediation applications.
\end{abstract}

\section{INTRODUCTION}

Triclocarban $\left(3,4,4^{\prime}\right.$-trichlorocarbanilide, TCC) is a synthetic broad-spectrum haloaromatic antimicrobial agent that has been extensively produced and employed in various household and personal care products since the 1950s. ${ }^{1}$ TCC primarily inhibits Gram-positive bacteria and is also partly active against Gramnegative bacteria as well as fungi. ${ }^{2}$ Worldwide, a large quantity of TCC is consumed per year and disposed into wastewater treatment plants (WWTPs). ${ }^{3}$ However, due to its poor water solubility $\left(0.65-1.55 \mathrm{mg} / \mathrm{L} \text { at } 25^{\circ} \mathrm{C}\right)^{1}$ and incomplete removal in WWTPs, approximately $3 \%$ of TCC remains in WWTP effluent and $75 \%$ of TCC is adsorbed in dewatered sludge., This incomplete removal opens a path for deposition of TCC into rivers, surface waters, sediments, farmlands, and forests in the form of contaminated effluents and sewage biosolids. In recent decades, numerous studies have reported the occurrence of TCC at up to $100 \%$ detection frequencies in various environments around the world including the United States and China, especially in WWTP effluents, surface waters, biosolids, and sediments (Tables S1 and S2). ${ }^{6-10}$ Specifically, sediments receiving treated and untreated wastewater inputs are known to contain TCC in high $\mu \mathrm{g} / \mathrm{kg}$ to low $\mathrm{mg} / \mathrm{kg}$ quantities. ${ }^{11}$ The average accumulative concentrations of TCC can reach up to $39 \pm 59$ and $25.2 \pm 0.6 \mathrm{mg} / \mathrm{kg}$ in tested biosolids from the United States and China, respectively. ${ }^{12,13}$ TCC has been ranked as one of the top 10 emerging environmental contaminants globally. ${ }^{1}$ Even its higher, lesser chlorinated and nonchlorinated congeners, such as $3,3^{\prime}, 4,4^{\prime}$-tetrachlorocarbanilide, 2,3',4,4'tetrachlorocarbanilide, 4,4'-dichlorocarbanilide (DCC), and

Received: September 27, 2016

Revised: November 17, 2016

Accepted: November 30, 2016

Published: November 30, 2016 
carbanilide (NCC) have also been simultaneously detected with TCC. ${ }^{7,14-16}$ Such multi-, and less-chlorinated carbanilides are also highly recalcitrant and poorly biodegradable both in aerobic and anaerobic environments. The estimated half-life of TCC in water, soil, and sediment is 60,120 , and 540 days, respectively. ${ }^{1,17}$

TCC residues in water have been found to be toxic to some aquatic organisms, such as algae, fish, and crustaceans. ${ }^{18}$ Through bioaccumulation along the food chain, TCC can be accumulated in the tissues of mammals and humans, resulting in endocrine disruption and potentially carcinogenic and genotoxic effects. ${ }^{1,11}$ The prevalence and persistence of TCC could also potentially lead to cross-resistance and the development of other antibiotics resistance genes. ${ }^{4}$ The reported transformation products of TCC, such as $\mathrm{N}$-hydroxylated and dechlorinated metabolites, also pose significant risks to human health and the environment. ${ }^{1}$ To eliminate these harmful effects of TCC and its hazardous byproducts, measures must be taken to remove these compounds from polluted environments. Biodegradation is a potential and economical approach for the removal of TCC and its corresponding metabolites. However, there are a limited number of studies on the biodegradation of TCC. In a simulated sewage system, $0.2 \mathrm{mg} / \mathrm{L}$ TCC was metabolized to chloroaniline intermediates and ultimately mineralized. ${ }^{19}$ An enrichment dominated by the members of the family Alcaligenaceae was able to use TCC or NCC as the sole carbon source. ${ }^{20}$ A triclosan-degrading strain of Sphingomonas sp. YL-JM2C, was found to be capable of degrading TCC to chloroanilines, which could be further mineralized. ${ }^{21}$ However, the molecular mechanism for the biodegradation of TCC, especially the key enzyme(s) responsible for the initial hydrolysis of the two amide bonds in TCC, has not been described.

The objectives of this study were to (i) isolate effective bacterial strain capable of biodegrading TCC and its dehalogenated congeners, DCC and NCC, (ii) identify the corresponding metabolic pathway, and (iii) clone functional genes associated with the biodegradation process and characterize the purified functional enzyme. We hypothesized that the initial step of TCC biodegradation was the two amide bonds hydrolysis, and the cleavage of the two amide bonds was mediated by an amidase. A genomic library screen was carried out to search for an amidase in the genome of the isolated TCCdegrading strain. Our results supported this hypothesis. The discovery of a novel TCC-hydrolyzing amidase fills a gap in the molecular basis of TCC biodegradation and provides a potential candidate for bioremediation applications.

\section{MATERIALS AND METHODS}

Chemicals, Bacterial Strains, and Culture Conditions. TCC (99\% purity), NCC (98\% purity), and high performance liquid chromatography (HPLC) grade acetonitrile were purchased from Sigma-Aldrich (St. Louis, MO, USA). DCC (98\% purity) was purchased from Fluorochem (Hadfield, UK). 3,4-Dichloroaniline (DCA, 98\% purity), 4-chloroaniline (4CA, 99\% purity), 3-chloroaniline (3CA, 99\% purity), aniline (AN), diflubenzuron ( $97 \%$ purity), propham (99\% purity), acetanilide (99\% purity), and 4-chloracetanilide (98\% purity) were purchased from Aladdin (Shanghai, China). All other chemicals used were of analytical grade. Required enzymes for DNA manipulation were all purchased from TaKaRa Biotech (Dalian, China). Stock solutions of TCC ( $1 \%, \mathrm{w} / \mathrm{vol})$, DCC ( $1 \%, \mathrm{w} / \mathrm{vol})$, and $\operatorname{NCC~}(1 \%, \mathrm{w} / \mathrm{vol})$ were prepared in dimethyl sulfoxide mixed with acetone $(1: 1 ; \mathrm{vol} / \mathrm{vol})$. Competent Escherichia coli
DH5 $\alpha$ and E. coli BL21 (DE3) (Tiangen Biotech, Beijing, China) were used in genome library construction and heterologous expression and cultured in Luria-Bertani (LB) medium at $37{ }^{\circ} \mathrm{C}$. Plasmid vectors pUC118 BamHI/BAP (Amp ${ }^{\mathrm{r}}$; TaKaRa, Dalian, China), pMD18-T (Amp ${ }^{\mathrm{r}}$; TaKaRa, Dalian, China), and pET-29a $(+)\left(\mathrm{Km}^{\mathrm{r}}\right.$; Novagen Co., Shanghai, China) were used for DNA cloning and gene expression. Mineral salt medium (MSM) consisted of $\mathrm{NaCl}(1 \mathrm{~g} / \mathrm{L}), \mathrm{NH}_{4} \mathrm{Cl}$ $(1 \mathrm{~g} / \mathrm{L}), \mathrm{K}_{2} \mathrm{HPO}_{4}(1.5 \mathrm{~g} / \mathrm{L}), \mathrm{KH}_{2} \mathrm{PO}_{4}(0.5 \mathrm{~g} / \mathrm{L})$, and $\mathrm{MgSO}_{4}$. $7 \mathrm{H}_{2} \mathrm{O}(0.2 \mathrm{~g} / \mathrm{L}), \mathrm{pH}$ 7.0. For solid media, $15 \mathrm{~g}$ agar was added per liter. The media were sterilized by autoclaving at $121{ }^{\circ} \mathrm{C}$ for $30 \mathrm{~min}$ before use. Ampicillin (Amp; $100 \mathrm{mg} / \mathrm{L}$ ) or kanamycin $(\mathrm{Km} ; 50 \mathrm{mg} / \mathrm{L})$ was supplemented to the media as necessary.

Isolation and Identification of TCC-Degrading Bacteria. Water and sediment samples (approximately $5 \mathrm{~g}$ ) from the Zhujiang River (Shenzhen, China) were used for enrichment with MSM $(50 \mathrm{~mL})$, supplemented with TCC $(63.4 \mu \mathrm{M})$ and yeast extract $(0.05 \%)$ in $150 \mathrm{~mL}$ flasks. Cultures were incubated on a rotary shaker at $30{ }^{\circ} \mathrm{C}$ and $150 \mathrm{rpm}$. After 7 days, $5 \mathrm{~mL}$ of enrichment culture was transferred to fresh media, and this process was repeated five times. Isolation was carried out by the dilution-to-extinction method on MSM agar plates amended with yeast extract $(0.05 \%)$ and TCC $(63.4 \mu \mathrm{M})$. Colonies with different colors and margins were streaked repeatedly on agar plates until pure cultures were obtained. The TCC-degrading activities of the cultures were further tested in MSM. TCCdegrading isolates were identified by morphological, physiological, biochemical, and 16S rRNA gene sequence analysis as described previously. ${ }^{22}$

Biotransformation Assays. Strain TCC-2 cultured to stationary phase in LB with TCC was harvested, washed, and resuspended to an optical density (OD) of 2.0 at $600 \mathrm{~nm}$ for inoculum. Aerobic biotransformation was performed in a $50 \mathrm{~mL}$ flask containing $20 \mathrm{~mL}$ of sterile MSM with a TCC concentration of $31.7 \mu \mathrm{M}$. The effect of $2 \mathrm{mM}$ glucose on TCC biotransformation was also investigated. For anaerobic hydrolysis of TCC, DCC, and NCC, sodium acetate $(15 \mathrm{mM})$ and potassium nitrate $(1.5 \mathrm{mM})$ were used as carbon source and electron donor and acceptor for growth support. Control cultures were not supplemented with potassium nitrate to test the hydrolysis ability of the initial inoculum. Anaerobic media $(80 \mathrm{~mL})$ in serum bottles $(100 \mathrm{~mL})$ were purged with nitrogen $(99.9 \%$ purity). The culture with $1.5 \%$ inoculum ( $\mathrm{vol} / \mathrm{vol}$ ) was incubated on a rotary shaker at $30^{\circ} \mathrm{C}$ and $150 \mathrm{rpm}$. No inoculum controls and those without substrates were also incubated under aerobic and anaerobic conditions. TCC and its corresponding transformation products were identified by ultraperformance liquid chromatography (UPLC)-tandem mass spectrometry (MS) and quantified by a reverse-phase HPLC as described below.

Chemical Analysis. TCC residues in water samples were extracted with an equal volume of dichloromethane. The organic phase was dried over anhydrous sodium sulfate and then evaporated at ambient temperature. Concentrated TCC residues were dissolved in $1 / 4$ volume acetonitrile. DCA, 4CA, and AN were monitored directly in water samples after filtration through $0.22 \mu \mathrm{m}$ filter. All samples were analyzed using a HPLC (model2695, Waters Co., Milford, MA, USA) equipped with a $C_{18}$ column $(5 \mu \mathrm{m}, 150 \mathrm{~mm} \times 4.6 \mathrm{~mm}$, Waters Co., Milford, MA, USA). The mobile phase was a mixture of acetonitrile and water $(60: 40 ; \mathrm{vol} / \mathrm{vol})$ at a flow rate of $0.8 \mathrm{~mL} / \mathrm{min}$ in isocratic mode. A UV detector (model 2487, Waters Co., Milford, MA, USA) was set at $275 \mathrm{~nm}$ for the detection of TCC and $240 \mathrm{~nm}$ for the detection of chloroanilines. AN was analyzed with mobile phase 
of methanol and water $(50: 50 ; \mathrm{vol} / \mathrm{vol})$ at a flow rate of $0.8 \mathrm{~mL} / \mathrm{min}$ under $288 \mathrm{~nm}$ as described previously. ${ }^{23}$ All data are represented as the means \pm standard deviation from at least three experimental measurements. Metabolites from the biotransformation of TCC and other amide compounds were identified using a Waters ACQUITY UPLC-MS system (Waters Co., Milford, MA, USA) equipped with a reverse phase $\mathrm{C}_{18}$ column (ACQUITY BEH $2.1 \times 50 \mathrm{~mm}, 1.7 \mu \mathrm{m}$; Waters Co., Milford, MA, USA) in gradient elution mode at a flow rate of $0.1 \mathrm{~mL} / \mathrm{min}$. The electrospray ionization source was used in positive mode, and parameters were set as follows: desolvation temperature, $350{ }^{\circ} \mathrm{C}$; gas flow, $900 \mathrm{~L} / \mathrm{h}$; and capillary voltage, $3 \mathrm{kV}$. The nebulizer and collision gas were both nitrogen.

Cloning and Sequence Analysis of the TCC-Hydrolyzing Amidase Gene (tccA). DNA manipulation and genomic library construction were carried out as described previously. ${ }^{24,25}$ Genomic DNA of strain TCC-2 was extracted as previously described. ${ }^{26}$ The genomic DNA was randomly digested with Sau3AI and fragments in the size range 4- to $8-\mathrm{kb}$ were pooled, ligated into the vector pUC118 BamHI/BAP and transformed into E. coli DH5 $\alpha$. Because TCC hydrolysis had no obvious selection marker, 4-acetaminophenol was used as the selection marker for the potential positive transformants on TCC. The colonies with brown color indicated the hydrolysis of the amide bond of 4-acetaminophenol to 4-aminophenol by potential amidases. ${ }^{24}$ Brown colonies were picked, cultured, and further tested for TCC amide bond hydrolysis in MSM and confirmed using UPLC-MS. One positive clone was selected and the inserted DNA fragment was sequenced by TaKaRa Biotech (Dalian, China). Nucleotide and deduced amino acid sequence analysis, open reading frame (ORF) determination, identity analysis, and multiple alignment of the amino acid sequence of TccA with other biochemically identified and putative amidases were performed as previously described. ${ }^{25}$ Phylogenetic analysis of TccA with homologous amidases was performed using MEGA (version 5.0). ${ }^{27}$ The final phylogenetic tree was polished by the Interactive Tree of Life (iToL) online software (http://itol.embl.de/). ${ }^{28}$

Gene Expression and Purification of the Recombinant Amidase. The amidase gene $t c c A$ was amplified from genomic DNA of strain TCC-2 with the primer pair of tccAF (5'-CGTCCTCCCGCATATGACTGCTACCGACGAGTTGTA-3'), in which an NdeI site was introduced and tccAR (5'-TATTCTCGAGCAGCGGCGGCTTGCGCGCCGCCCAGGGATG-3'), in which an XhoI site was introduced. The PCR product of $t c c A$ was subcloned into linearized pMD18-T vector and digested with NdeI and XhoI. The plasmid pET$29 \mathrm{a}(+)$ was also digested with $\mathrm{NdeI}$ and XhoI, and then ligated with the digested PCR product to generate the recombinant plasmid pET29a-tccA. The recombinant plasmid was transformed into competent E. coli BL21(DE3). Detailed induction and purification procedures for TccA were the same as those described previously. ${ }^{29}$ The purity and molecular mass of the denatured TccA was evaluated by SDS-PAGE. The molecular mass of the native TccA was determined using an Ultraflex III matrix-assisted laser desorption-ionization time-of-flight mass spectrometer (MADIL-TOF-MS, Bruker Co., ReflexIII, Germany) equipped with a solid-state smart beam, using FlexControl v3.3 software (Bruker Daltonics $\mathrm{GmbH}$ ). Protein concentration was measured using the BCA Protein Assay Kit (Sangon Biotech, Shanghai, China) with bovine serum albumin as the standard. The isoelectric point ( $\mathrm{pI}$ ) of TccA was estimated by PAGE with $6.25 \%$ Ampholine ( $\mathrm{pH} 3.5$ to 10$)$ in a gel rod $(0.5$ by $1.0 \mathrm{~cm})$ using an isoelectric focusing kit for calibration (Pharmacia LKB Biotechnology) according to the manufacturer's instructions.

Enzyme Assay. The activity of purified TccA was measured in $50 \mathrm{mM}$ PBS ( $\mathrm{pH}$ 7.0) supplemented with different substrates. Controls without TccA were also carried out under the same conditions. The reaction conditions were $35{ }^{\circ} \mathrm{C}$ for $30 \mathrm{~min}$, and the reaction was terminated by the addition of $5 \mu \mathrm{L}$ of $\mathrm{HCl}(5.0 \mathrm{M})$ and immediately kept on ice. One unit of enzyme activity was defined as the amount of enzyme required to catalyze the formation of $1 \mu \mathrm{mol}$ product per minute. To determine whether $t c c A$ was constitutively transcribed in strain TCC-2, cells cultured in LB broth and in LB broth supplemented with TCC, DCC, or NCC $(31.7 \mu \mathrm{M})$ were collected and treated with an ultrasonic cell crusher (JY92-IIDN, Ningbo SCIENTZ Biotech Co., Ltd., China) to obtain crude bacterial lysate. The hydrolytic activity of these crude enzymes was compared in $50 \mathrm{mM}$ PBS ( $\mathrm{pH}$ 8.0) supplemented with the corresponding substrates $(31.7 \mu \mathrm{M})$ and incubated at $35{ }^{\circ} \mathrm{C}$ for $30 \mathrm{~min}$ in triplicate. Differences between the two treatments were statistically analyzed by the two-tailed unpaired $t$ test.

Biochemical Characterization of TccA. To test the thermal stability of TccA, the enzyme was preincubated in $50 \mathrm{mM}$ PBS ( $\mathrm{pH} \mathrm{7.0)}$ ) at temperatures of $15,20,25,30,35,40$, $50,60,70$, and $80^{\circ} \mathrm{C}$ for $1 \mathrm{~h}$. Following the incubation period, the reaction systems were supplemented with TCC $(31.7 \mu \mathrm{M})$ and incubated at $35{ }^{\circ} \mathrm{C}$ for $30 \mathrm{~min}$. For determination of the optimal temperature, TCC hydrolysis was performed at the same temperature range for $30 \mathrm{~min}$. To test the acid-alkali stability of TccA, different buffer solutions, including $50 \mathrm{mM}$ citric acid-sodium phosphate ( $\mathrm{pH} 4.0$ to 6.0 ), $50 \mathrm{mM} \mathrm{KH}_{2} \mathrm{PO}_{4}-$ $\mathrm{K}_{2} \mathrm{HPO}_{4}$ buffer ( $\mathrm{pH} 6.0$ to 8.0 ), and $50 \mathrm{mM}$ glycine- $\mathrm{NaOH}$ $(\mathrm{pH} 8.5$ to 10.0$)$ were prepared. TccA was preincubated in different $\mathrm{pH}$ buffers at $4{ }^{\circ} \mathrm{C}$ for $24 \mathrm{~h}$ and then incubated at $35^{\circ} \mathrm{C}$ for $30 \mathrm{~min}$. Measurement of the optimal $\mathrm{pH}$ was similar to that of optimal temperature. The effect of various metal ions, chemical detergents, and inhibitors was also determined by adding them to the reaction systems at $35{ }^{\circ} \mathrm{C}$ for $30 \mathrm{~min}$. TccA-free and compound-free negative controls were performed and the relative activity was determined by comparison to TccA activity without any potential inhibitors. Each reaction was carried out in triplicate.

Total RNA Preparation and RT-qPCR. Strain TCC-2 was cultured in LB medium supplemented with TCC, DCC, or NCC $(31.7 \mu \mathrm{M})$. Cultures grown in the absence of the above substrates were used as controls. At the late-exponential phase, total RNA was extracted using the RNAprep Cell/Bacteria Kit (Tiangen Biotech, Beijing, China) following the manufacturer's protocol. DNA was erased with recombinant DNase I (RNasefree, TaKaRa Biotech, Dalian, China) and cDNAs were synthesized with PrimeScript RTase (TaKaRa Biotech, Dalian, China). cDNAs were then used as templates for the RT-qPCR amplification using the fast real-time PCR system (Applied Biosystems7500, USA) with SYBR Green Premix (TaKaRa Biotech, Dalian, China) and RT-qPCR primers of RTtccAF (5'-GCCCACCTCGTACGAGTATT-3') and RTtccAR ( $5^{\prime}$-GAAGATGCCGTCCAGACCAT-3'). Data were analyzed using the comparative threshold cycle $\left(C_{\mathrm{T}}\right)$ method $^{30}$ with the internal standard $16 \mathrm{~S}$ rRNA gene as the reference. The primer pair was 16S-F(5'-GACGGTAACCGGAGAAGAAG-3') and 16S-R(5'-CGTGGACTACCAGGGTATCT-3'). Three biological and three technical replicates were performed for each sample.

Nucleotide Sequence Accession Numbers. The $16 \mathrm{~S}$ rRNA gene and the DNA fragment $(6,475 \mathrm{bp})$ containing the amidase gene $t c c A$ from Ochrobactrum sp. TCC-2 have been 
deposited in GenBank database under accession numbers KT820193 and KU753911, respectively.

\section{RESULTS}

Strain Isolation and Identification. The samples from the Zhujiang River in China were serially passaged in enrichment media containing TCC. After the fifth transfer, 4CA and DCA were detected in the TCC-degrading enrichment culture. By dilution spread, a relatively homogeneous collection of colonies was seen on plates. One of the isolates, named TCC-2, was selected for further study for its higher TCC hydrolyzing ability. Strain TCC-2 was Gram-negative, short rod-shaped, and motile with a polar flagellum bacterium (Figure S1). Its colonies were milky white, circular, and convex after overnight incubation on LB agar. The $16 \mathrm{~S}$ rRNA gene sequence of strain TCC-2 exhibited $100 \%, 99.3 \%$, and $99.3 \%$ sequence similarity with those of O. lupini LUP21 ${ }^{\mathrm{T}}$ (AY457038), O. anthropi ATCC $49188^{\mathrm{T}}$ (CP000758) and O. cytisi $\mathrm{ESC1}^{\mathrm{T}}$ (AY776289), respectively. A phylogenetic tree based on all known validly described representatives of the genus Ochrobactrum is shown in Figure S2. On the basis of the above data, strain TCC-2 was preliminarily identified as Ochrobactrum sp.

Aerobic Hydrolysis of TCC and Identification of Metabolites. As shown in Figure 1A, when glucose was added to the cultures, $96.88 \pm 0.05 \%$ of the initial amount of TCC $(31.7 \mu \mathrm{M})$ was hydrolyzed by strain TCC- 2 within $24 \mathrm{~h}$, which was higher than the cultures without glucose (76.92 \pm $8.64 \% ; P=0.057)$. This could be attributed to an increase in the biomass of strain TCC-2 when glucose was supplied, which significantly increased the TCC hydrolysis rate $(0.112 \pm$ $\left.0.014 \mathrm{~h}^{-1}\right)$ compared to no glucose supply $\left(0.066 \pm 0.010 \mathrm{~h}^{-1}\right.$; $P=0.007)$. Strain TCC-2 was unable to use TCC as the sole carbon and energy source as evidenced by the lack of change in optical density (Figure 1C). The two main metabolites were identified as 4CA and DCA by comparison of characteristic peaks with the corresponding authentic standards by HPLC and confirmed by UPLC-MS. At retention times of 4.36 and $5.77 \mathrm{~min}, 4 \mathrm{CA}$ exhibited a prominent protonated molecular ion at $\mathrm{m} / z$ of 128 and $130[\mathrm{M}+\mathrm{H}]^{+}$isotope clusters for one chlorine atom, while DCA had a prominent protonated molecular ion at $m / z$ of 162,164 , and $166[\mathrm{M}+\mathrm{H}]^{+}$isotope clusters for two chlorine atoms. These results were consistent with the mass spectral features of the authentic standards. Meanwhile, 4CA and DCA could not be detected in the no inoculum control (Figure 2). Taken together, these results demonstrated that strain TCC-2 was able to hydrolyze the two amide bonds of TCC to generate 4CA and DCA. After $72 \mathrm{~h}$, approximately $81 \%$ of the theoretically expected 4CA had accumulated in the MSM regardless of the presence or absence of glucose. The formation rates were $0.026 \pm 0.006$ and $0.026 \pm 0.003 \mathrm{~h}^{-1}$ for groups without and with glucose, respectively. Meanwhile, $53.45 \pm 2.89 \%$ and $50.78 \pm 3.31 \%$ of the theoretically expected DCA was formed in MSM with and without glucose, respectively. The corresponding formation rates were $0.009 \pm 0.001$ and $0.009 \pm 0.002 \mathrm{~h}^{-1}$. The formation rate constants of 4CA $(P=0.020)$ and DCA $(P=0.057)$ in cultures containing glucose were both significantly higher than those without glucose within the initial $10 \mathrm{~h}$ and displayed negligible differences within $72 \mathrm{~h}$ (Table S3).

Anaerobic Hydrolysis of TCC under the Nitrate Respiration Condition. Colonization of the introduced TCCdegrading bacteria in TCC-contaminated anoxic environments is an important prerequisite for effective bioremediation.

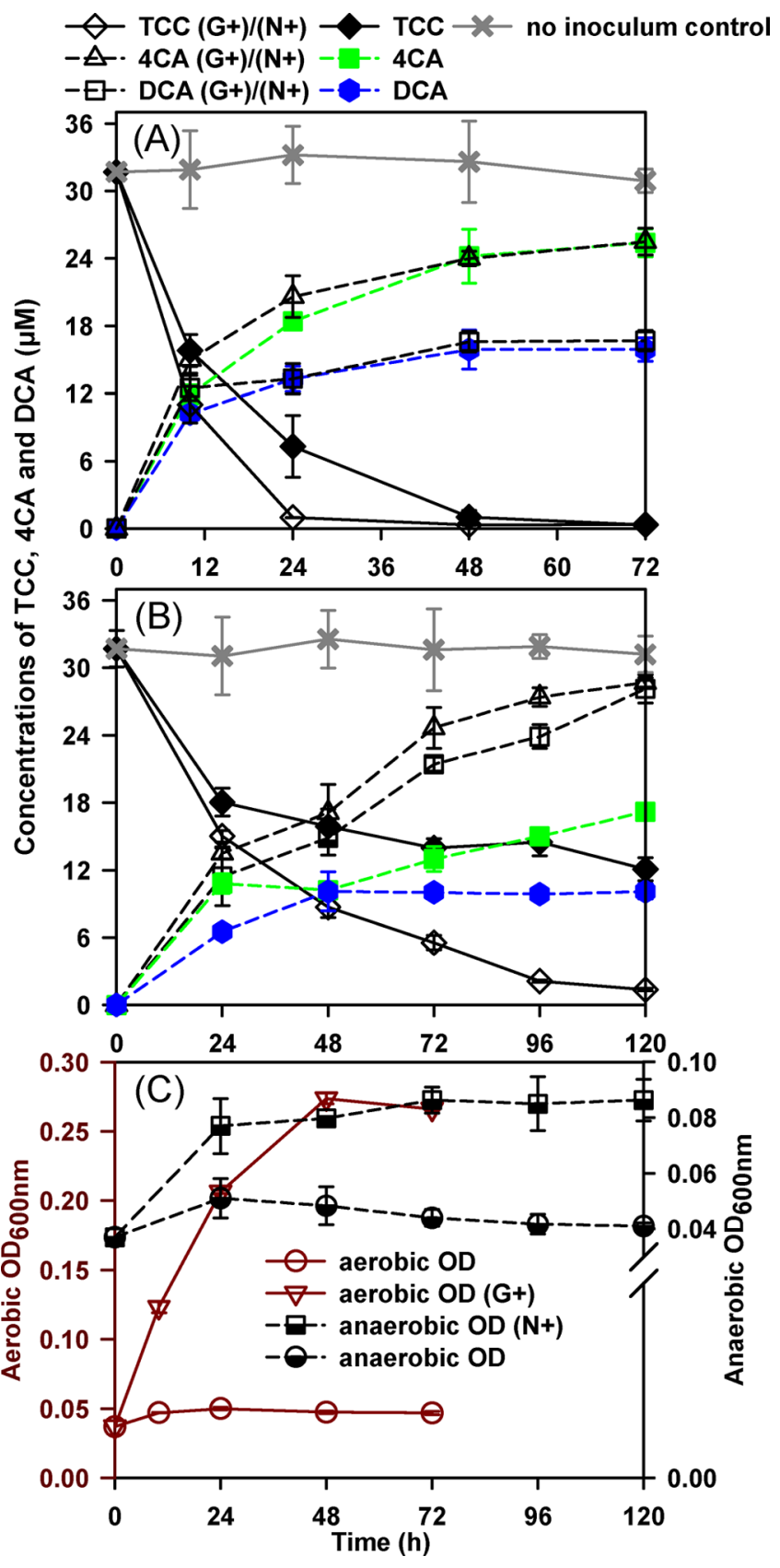

Figure 1. Aerobic biotransformation of TCC bystrain TCC-2 in the presence $(\mathrm{G}+)$ or absence of glucose (A). Anaerobic biotransformation of TCC by strain TCC- 2 in the presence $(\mathrm{N}+)$ or absence of nitrate (B). Growth of strain TCC-2 under aerobic conditions with $(\mathrm{G}+)$ or without glucose and under anaerobic conditions with $(\mathrm{N}+)$ or without nitrate $(\mathrm{C})$. The data are represented as the mean \pm standard deviation for triplicate incubations.

In this study, anaerobic hydrolysis of TCC was carried out with strain TCC-2 under the nitrate respiration condition. The basis was that the genome of strain TCC- 2 contains a complete set of genes for denitrification (data not shown) and nitrate is a thermodynamically favorable electron acceptor for anaerobic respiration for bioremediation in anoxic environments. ${ }^{31}$ As shown in Figure 1 panels B and C, strain TCC-2 could grow and convert TCC to 4CA and DCA under anaerobic conditions when nitrate was provided. Nearly $100 \%$ nitrate-N was rapidly reduced to nitrite-N within $24 \mathrm{~h}$ with a short period of nitrite-N accumulation (from $63.19 \pm 0.76 \%$ at the 16 th $\mathrm{h}$ to $36.73 \pm$ 

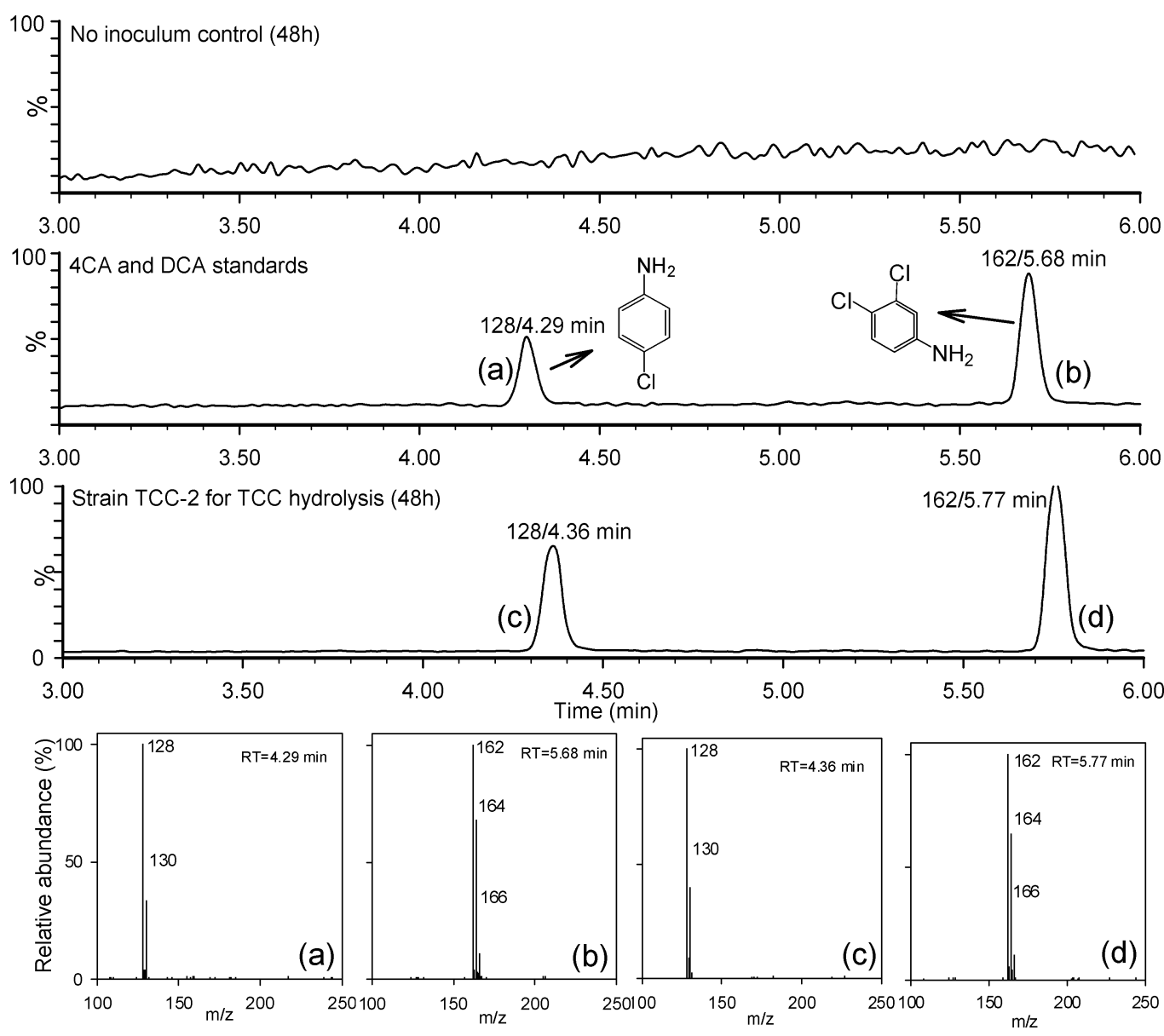

Figure 2. TIC and mass spectra profiles of 4CA and DCA standards as well as TCC hydrolysis products by the inoculation of strain TCC-2 (at $48 \mathrm{~h}$ ). The generated products with the retention times of 4.36 and $5.77 \mathrm{~min}$ by strain TCC-2 (at $48 \mathrm{~h}$ ) were identified as 4CA and DCA according to the same $\mathrm{m} / z$ and characteristic isotope clusters between them (cd) and standards (ab). No inoculum TCC hydrolysis control did not generate 4CA and DCA products at $48 \mathrm{~h}$ as shown by the TIC profile.

$6.82 \%$ at the 24th $\mathrm{h}$ ) and completely exhausted without accumulation of nitrite- $\mathrm{N}$ within $48 \mathrm{~h}$. The biomass was obviously increased in this period. Also, $72.70 \pm 2.90 \%$ of the initially supplied TCC $(31.7 \mu \mathrm{M})$ was hydrolyzed by strain TCC-2, in contrast to $50.08 \pm 4.89 \%$ of the control without nitrate supply $(P=0.005)$. After $120 \mathrm{~h}, 95.68 \pm 0.34 \%$ and $61.98 \pm 3.19 \%$ of TCC was hydrolyzed for groups with and without nitrate with rates of $0.027 \pm 0.001$ and $0.007 \pm$ $0.002 \mathrm{~h}^{-1}$, respectively. Correspondingly, more than $90 \%$ of the theoretically expected 4CA and DCA were accumulated under the nitrate respiration condition, both of which were significantly higher than those from the control group $(P<0.005)$. The increased biomass of strain TCC-2 under the denitrifying condition enhanced the anaerobic hydrolysis of TCC, which was indicated by the significantly increased formation rates for 4CA and DCA (Table S3). Additionally, the dehalogenated congeners of TCC, NCC, and DCC had transformation efficiencies of $91.19 \pm 2.58 \%$ at $48 \mathrm{~h}$ and $82.89 \pm 1.87 \%$ at 80 h, respectively (Figure S3).

Gene Cloning and Sequence Analysis. Out of approximately 10000 transformants, five brown colonies were selected due to the hydrolysis of the amide bond of 4-acetaminophenol. These colonies were further tested for growth and hydrolysis activity in LB and MSM media containing 4-acetaminophenol or TCC. As a result, only one colony, named U135, was capable of hydrolyzing the two amide bonds of TCC to generate 4CA and DCA. The cloned fragment was sequenced and was found to be $6475 \mathrm{bp}$, with six complete ORFs identified using OMIGA 2.0 software. On the basis of BLASTP analysis, one 1425 bp ORF shared homology with many biochemically characterized and putative amidases. This ORF along with its upstream sequence (200 bp) was subcloned into the vector pMD18-T and transformed into E. coli DH5 $\alpha$. A positive transformant was screened and was found to hydrolyze $85.01 \pm 3.69 \%$ of the supplemented TCC $(31.7 \mu \mathrm{M})$ within $48 \mathrm{~h}$. The metabolites were identified as 4CA and DCA as mentioned above. Consequently, the 1425 bp ORF was confirmed to be the target gene involved in the hydrolysis of the two amide bonds of TCC and designated as tccA (triclocarban-hydrolyzing amidase gene).

On the basis of sequence analysis, $t c c A$ had a G+C content of $67.9 \%$ and encoded a protein of 474 amino acids. A putative Shine-Dalgarno (SD) sequence was found $11 \mathrm{bp}$ upstream of the ATG start codon (CGAGAGGAACGGGCCCCTAATG, underlined). The deduced TccA protein shared low amino acid sequence identity ( 27 to $38 \%$ ) with several biochemically characterized amidases, including o-nitroacetanilide and $\omega$-lactams hydrolase OctHD (38\% identity; GenBank accession number BAI44731), p-nitroacetanilide hydrolase Aam (36\% identity; K9NBS6), 6-aminohexanoate-cyclic-dimer hydrolase NylA (35\% identity; P13397), linuron hydrolase LibA (34\% identity; AEO20132), propyzamide hydrolase 
CamH (33\% identity; KM243930), propanil hydrolase PamH (31\% identity; JF919485), 2,6-dichlorobenzamide, o-chlorobenzamide, and benzamide hydrolase BbdA ( $31 \%$ identity; KP792998), aryl acylamides ( $p$-nitroacetanilide, $p$-acetaminophenol, phenacetin, 4-chloroacetanilide and acetanilide) hydrolase AAA (31\% identity; FJ755834), aryl acylamides (2-chlorophenylacetamide, 2-phenylpropionamide and 2-methyl3-phenylpropionamide) hydrolase AmdA (30\% identity; AAK11724) and amide pesticides hydrolase AmpA (preferred substrates including propanil, propham, and chlorpropham, 27\% identity; JQ388838). Multiple alignment of the amino acid sequence of TccA with the biochemically characterized amidases revealed high conservation of the Gly/Ser-rich motif (GGSS[GS]G) and the catalytic triad (Ser-Ser-Lys) of the amidase signature enzyme family ${ }^{32}$ (Figure S4).

Gene Expression and Purification of $\mathrm{His}_{6}$-Tagged TccA. The $t c c A$ fragment was cloned into the vector pET-29a $(+)$ and transformed into E. coli BL21(DE3). One positive transformant was selected and its ability to degrade TCC was confirmed. 4CA and DCA were continually accumulated from TCC hydrolysis within $72 \mathrm{~h}$ (Figure S5). After IPTG induction, the expressed protein was purified from the crude extract using Ni-nitrilotriacetic acid affinity chromatography. TccA catalyzed the hydrolysis of TCC to equimolar amounts of 4CA and DCA (Figure S6). The purified, denatured protein showed a single band on SDS-PAGE, with a molecular mass of approximately $50 \mathrm{kDa}$, which was in agreement with the calculated molecular mass of $50000.63 \mathrm{Da}$. The experimentally determined molecular mass of the native TccA was 50733 Da by MALDI-TOF/ MS, indicating the enzyme was a monomer (Figure S7). The pI value of TccA was estimated to be 5.08 .

Transcription Response of tccA to Different Substrates. TCC and its dehalogenated congeners DCC and NCC were hydrolyzed by TccA, therefore, we determined the transcriptional level of $t c c \mathrm{~A}$ in strain TCC-2 in response to the above substrates by RT-qPCR. As shown in Figure 3A, $t c c A$ was constitutively transcribed and its expression was not obviously enhanced by NCC, DCC, and TCC, when compared with cells grown in LB medium alone. This was in accordance with the corresponding accumulation of their products, except that DCA production from TCC hydrolysis was significantly different $(P<0.05)$ (Figure $3 \mathrm{~B})$.

Characteristics of TCCA. The optimal temperature and $\mathrm{pH}$ value for TccA activity was $35^{\circ} \mathrm{C}$ and 8.0, respectively. Approximately $50 \%$ activity was retained when TccA was incubated at $60{ }^{\circ} \mathrm{C}$ for $1 \mathrm{~h}$, while TccA was inactivated at temperatures above $70{ }^{\circ} \mathrm{C}$. When the $\mathrm{pH}$ was 4.0 or less, less than $50 \%$ activity was retained. TccA could tolerate a $\mathrm{pH}$ value as high as 10.0. Even incubating TccA at $\mathrm{pH} 10.0$ for $24 \mathrm{~h}$, greater than $80 \%$ activity was retained (Figure 4). TccA activity was negligibly affected by $1 \mathrm{mM}$ of $\mathrm{Mg}^{2+}, \mathrm{Ni}^{2+}, \mathrm{Li}^{+}$, and $\mathrm{Zn}^{2+}$. Other tested metal ions reduced enzyme activity by less than $20 \%$. However, TccA was completely inhibited by $1.0 \mathrm{mM}$ $\mathrm{Ag}^{+}$(Table S4). The surfactants Triton X-100 (10 mM) and SDS ( 1 or $10 \mathrm{mM}$ ) also had negligible effects on TccA activity, however, Tween-80 (10 mM) inhibited TccA activity by approximately $50 \%$. EDTA and the sulfhydryl reagent iodoacetamide only slightly inhibited TccA activity. This suggested that no sulfhydryl group existed in the active sites of TccA. Histidine modifier diethyl pyrocarbonate (DEPC) and chelator 1,10-phenanthroline also decreased TccA activity between 40 and $50 \%$. Nearly $40 \%$ activity reduction of TccA by the serine protease inhibitor phenylmethylsulfonyl fluoride
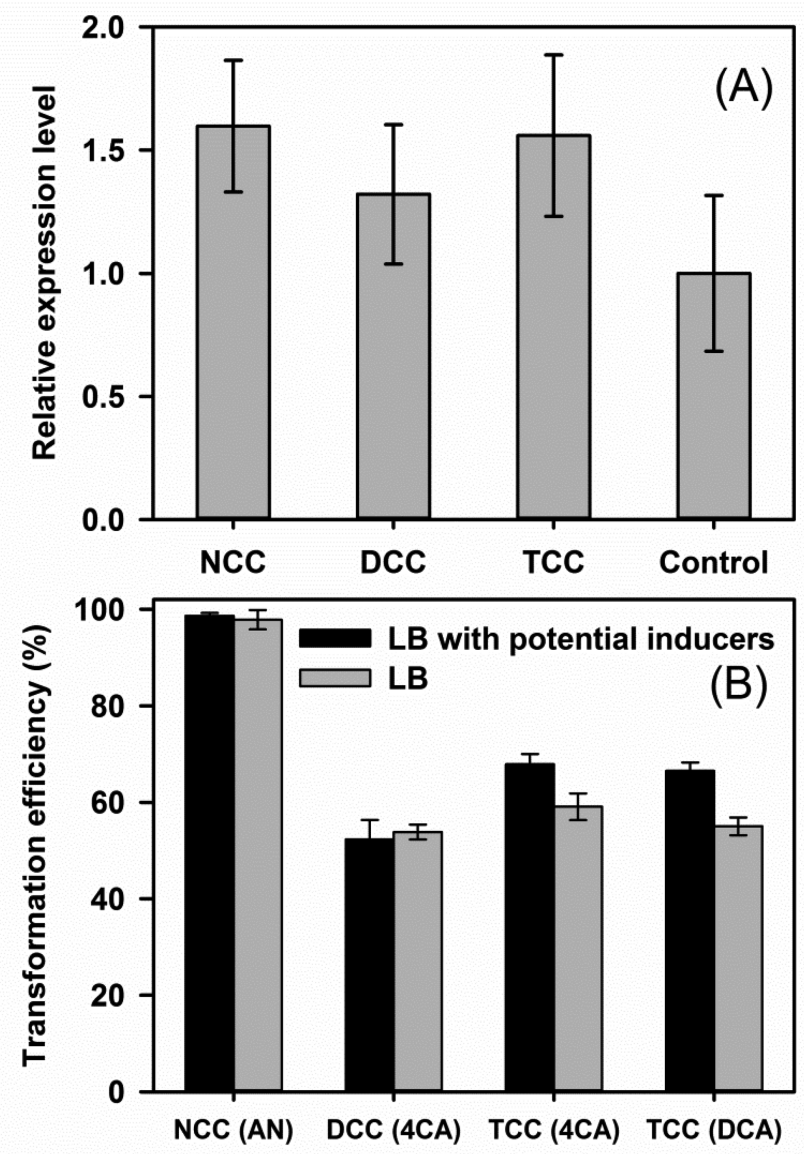

Figure 3. RT-qPCR analysis of the transcription of $t c c A$ in strain TCC-2 in the presence of NCC, DCC, and TCC (A). Gene expression was compared with the LB media control and 16S rRNA gene was used as an internal control. Hydrolysis activity of crude bacterial lysates from strain TCC-2 cultured in LB, LB+NCC, $\mathrm{LB}+\mathrm{DCC}$, or $\mathrm{LB}+\mathrm{TCC}$ media (B).

(PMSF) may be due to the presence of two serines in the active sites of TccA.

Substrate Spectrum and Specificity of TccA. The activity of TccA to hydrolyze some insecticides, herbicides, and chemical intermediates was determined as well. As shown in Table S5, compared with TCC, DCC, and NCC, TccA transformed diflubenzuron at a relatively low rate and formed 2,6-difluorobenzoic acid and 4CA as identified by UPLC-MS (Figure S8). 2,6-Difluorobenzoic acid was also the product of diflubenzuron hydrolyzed by ampA. However, the other amide bond of diflubenzuron was maintained and formed 4-chlorophenylurea. ${ }^{24}$ TccA was also able to hydrolyze the amide bond of propham and chlorpropham and accumulated $\mathrm{AN}$ and 3CA (Figure S8), while meta-chlorinated propham had a specially low reaction rate. This may be due to the chlorinated site, another herbicide barban, which also had a predicted 3CA product, was not hydrolyzed by TccA. For monochlorophenylurea, dichlorophenylurea, bromophenylurea, and fluorophenylurea, which were important intermediates for chemical synthesis, the amide bond in these compounds were also hydrolyzed except that of the fluorinated phenylurea. 4-Chlorophenylurea and 1-(3,4-dichlorophenyl)urea had comparable specific activity values, and both were higher than that of 4-bromophenylurea (Table S5 and Figure S8). The hydrolysis of acetanilide by TccA was obviously weaker than its chlorinated congener 

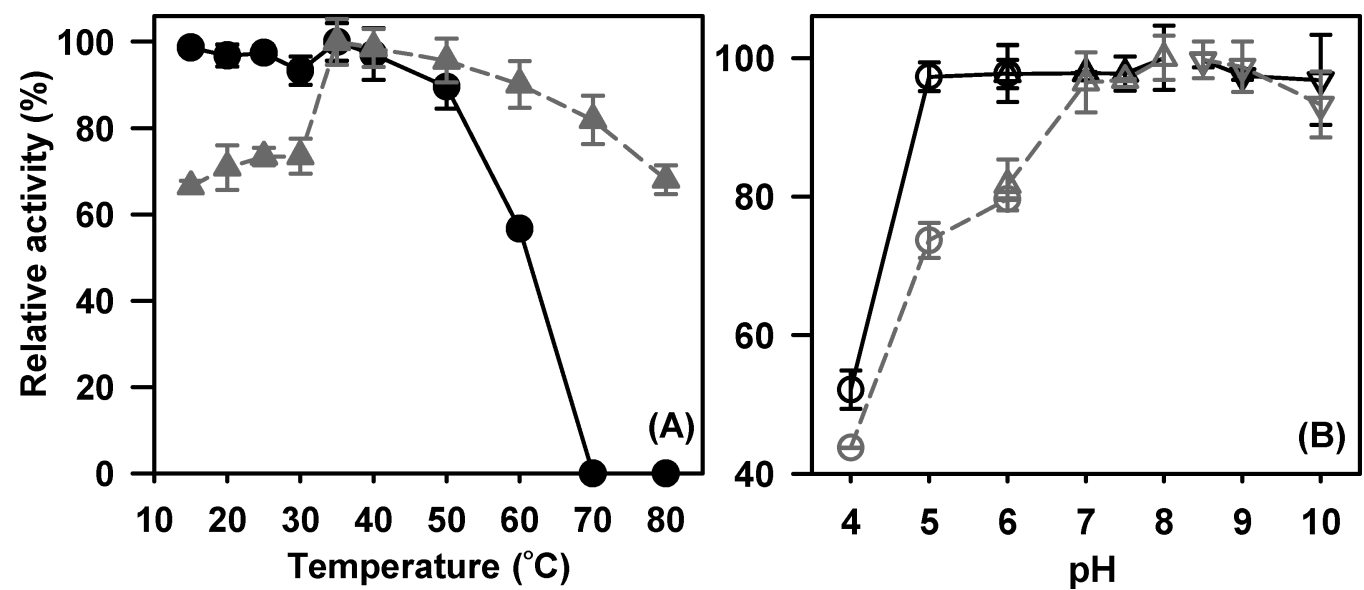

Figure 4. Effect of temperature $\left(15\right.$ to $80{ }^{\circ} \mathrm{C}$ ) on the stability (continuous line) and activity (dashed line) of TccA (A). Effect of $\mathrm{pH}$ (4 to 10$)$ on the stability (continuous line) and activity (dashed line) of TccA (B). $50 \mathrm{mM}$ citric acid-sodium citrate (pH 4 to $6 ; 0$ ); $50 \mathrm{mM} \mathrm{KH}_{2} \mathrm{PO}_{4}-\mathrm{K}_{2} \mathrm{HPO}_{4}$ buffer (pH 6 to $8 ; \triangle)$; $50 \mathrm{mM}$ glycine- $\mathrm{NaOH}(\mathrm{pH} 8.5$ to $10 ; \nabla)$.

4-chloracetanilide. The substituent group on the benzene ring seemed to determine the steric bond with the active site of TccA. Interestingly, AN and 4-amino-2-chloropyridine were detected for the hydrolysis of forchlorfenuron, which contained a structure of heterocycle. This illustrated that both of the two amide bonds were hydrolyzed and the specific activity was $19.04 \pm 0.92$ (Table S5 and Figure S8). It is interesting to note that the amide bond from the structure of benzamide or within the side chain was unfavorable for TccA, which had no activity for such substrates such as benzamide, 2,6-dichlorobenzamide (BAM) propyzamide, and antibiotics chloramphenicol and florfenicol. TccA showed 31\% homology with the reported amidase BbdA, which was responsible for the degradation of BAM. ${ }^{33}$

\section{DISCUSSION}

The extensive use and insufficient treatment of TCC in WWTPs has led to its accumulation in various environments, especially in sediments and biosolids, in which TCC concentrations have been reported up to $\mathrm{mg} / \mathrm{kg}$ levels. ${ }^{12,13,15}$ Though the food and drug administration (FDA) recently passed a ban on TCC in soaps (http://www.fda.gov/NewsEvents/Newsroom/ PressAnnouncements/ucm517478.htm), for agricultural soils irrigated with WWTP secondary effluents or with biosolids application, TCC still has been frequently detected. The fate of TCC in different environmental matrices following these inputs is not yet completely understood. ${ }^{34}$ Thus, effective measures must be taken to avoid its reintroduction into the environment and to handle its spread. Biodegradation is considered a potential and economical method for the removal of this contaminant. Isolation of diverse TCC-degrading bacteria is the basic foundation for potential bioremediation applications. However, TCC-degrading strains have rarely been reported because of its recalcitrance and antimicrobial characteristics. ${ }^{1,16}$ So far, only one pure culture, Sphingomonas sp. YL-JM2C, has been isolated and shown to mineralize low concentrations of TCC by the initial hydrolysis of the two amide bonds of TCC. ${ }^{21}$ Therefore, in this study, the isolation of a new TCC-degrading strain, Ochrobactrum sp. TCC-2, provides another candidate for potential TCC bioremediation. It has been reported that Ochrobactrum sp. can degrade several organic pollutants, such as chlorothalonil, vinyl chloride, pyrethroids, polychlorinated biphenyls, and nicotine. ${ }^{35-39}$ However, this is the first report of an Ochrobactrum sp. that can degrade TCC.
Enzymes involving in the degradation of TCC have not been reported or characterized previously. Strain TCC-2 was incapable of mineralizing TCC but converted TCC to 4CA and DCA. The TCC-hydrolyzing amidase, TccA, responsible for the initial hydrolysis of the two amide bonds of TCC, was systematically analyzed. To the best of our knowledge, TccA is the first reported amidase that can catalyze the hydrolysis of TCC. On the basis of the sequence alignment and phylogenetic analysis, TccA shares low homology (27 to $38 \%$ ) with other biochemically characterized amidases (available from NCBI Swissprot protein database) (Figure 5). TccA formed an independent lineage with several putative amidases (available from NCBI nonredundant protein sequences database) belonging to Streptomyces (coverage 98 to $99 \%$; 51 to $53 \%$ identity) and Nocardiopsis (coverage 91\%; 44\% identity) species. Interestingly, multiple sequence alignment of TccA with all available putative amidases from Streptomyces and Nocardiopsis species (in total 18; coverage 91 to $99 \%$; 44 to $53 \%$ identity) revealed the presence of a highly conserved Gly/Ser-rich motif. But the first glycine was replaced by an alanine in these putative amidases, which was a little different and imperfect compared to that in TccA and other characterized members of the amidase signature enzyme family (Figures S4 and S9). TccA showed the highest similarity (coverage $96 \% ; 38 \%$ identity) with the biochemically characterized $o$-nitroacetanilide and $\omega$-lactams-hydrolyzing amidase OctHD from Rhodococcus sp. Oct $1 .^{40}$ Further analysis of these multiple alignments showed that the Gly/Ser-rich motif of OctHD (GGSSGA) was different from that in TccA and other biochemically characterized amidases (GGSS[GS]G) (Figures S4 and S9).

Previously identified amidases, such as LibA, PamH, and AmpA, are capable of hydrolyzing the chlorinated amide pesticides propanil and linuron to produce DCA, ${ }^{24,41,42}$ which was also the hydrolysis product of TCC by TccA. However, TccA showed low homology (27 to $34 \%$ identity) to these three amidases. AmpA hydrolyzes only one side of the two amide bonds of diflubenzuron to generate 2,6-difluorobenzoic acid and 4-chlorophenylurea but not 4CA, ${ }^{24}$ indicating AmpA cannot attack the amide bond of the 4-chlorophenylurea side. Interestingly, TccA was able to hydrolyze the two amide bonds of diflubenzuron to produce 2,6-difluorobenzoic acid and 4CA. Moreover, we found that TccA could also hydrolyze the two amide bonds of the dehalogenated congeners of TCC, DCC, and $\mathrm{NCC}$, to $4 \mathrm{CA}$ and $\mathrm{AN}$, respectively (Figure $\mathrm{S} 3$ and 


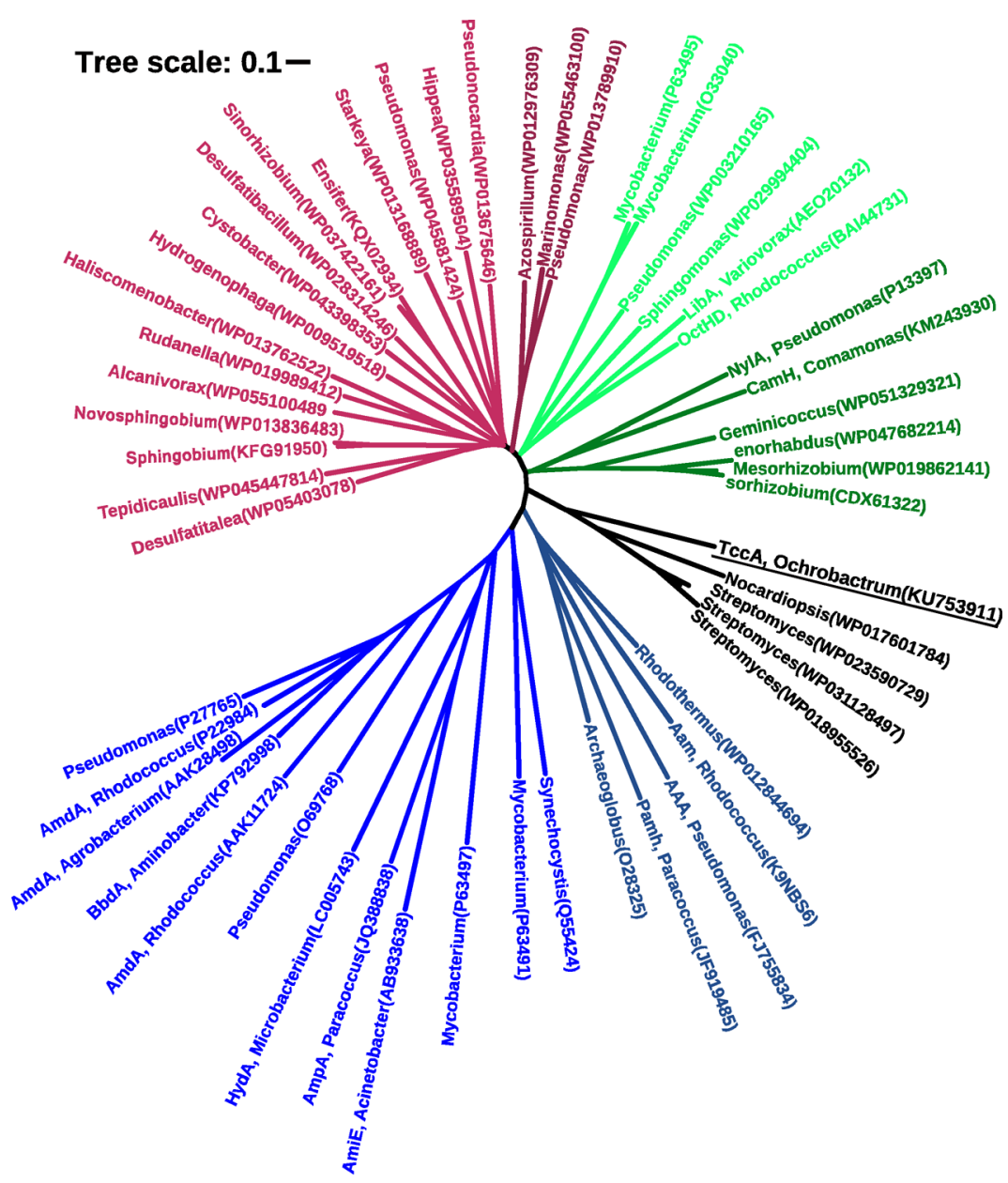

Figure 5. Phylogenetic tree constructed by the neighbor-joining method based on TccA and 52 homologous amidases from different hosts. Both the biochemically characterized (15) and putative amidases (37) were aligned with TccA (black and underlined). The 15 biochemically characterized amidases are as follows: AmdA (2-phenylpropionamide hydrolase from Agrobacterium tumefaciens strain d3, AAK28498); AmdA (propionamide hydrolase from Rhodococcus erythropolis N-774, P22984); unnamed amidase (propionamide hydrolase from Pseudomonas chlororaphis B23, P27765); HydA (acylhydrazides hydrazidase from Microbacterium sp. HM58-2, LC005743); AmiE (N-acyl-L-homoserine lactones hydrolase from Acinetobacter sp. Ooi24, AB933638); and OctHD (o-nitroacetanilide and $\omega$-lactams hydrolase from Rhodococcus sp. Oct1, BAI44731). See Figure S4 legend for provenance of LibA, PamH, BbdA, CamH, AAA, AmpA, Aam, NylA, and AmdA (AAK11724). The scale bar represents 0.1 substitutions per site, and GenBank accession numbers are in parentheses.

Table S5). DCC and NCC have also been frequently detected in sediments and biosoilds as the reductive dehalogenation of TCC dominates in anaerobic environments. ${ }^{7,8,14-16,43}$ TccA is capable of maintaining activity and hydrolyzing TCC over a wide range of $\mathrm{pH}$ and temperature values. Even kept at $\mathrm{pH} 10.0$ for $24 \mathrm{~h}$ or $60{ }^{\circ} \mathrm{C}$ for $1 \mathrm{~h}$, more than $80 \%$ and approximately $50 \%$ activity was retained, respectively. Furthermore, TccA was insensitive to many chemical inhibitors and metal ions. The stability of TccA meant its high tolerance to harsh conditions. Additionally, unlike the amidase HydA, which was induced by the substrate acylhydrazide, ${ }^{44}$ TccA as well as other reported amidases, such as BbdA, LibA, and AmpA ${ }^{24,33,42}$ were constitutively expressed. This suggests that $t c c A$ will be expressed under all conditions and not require TCC induction, making this strain useful for low-dose polluted sites. Wide range of substrate spectrum of TccA would be beneficial for bioremediation applications. Bioaugmentation with biodegradative bacteria for the bioremediation of emerging environmental micropollutants have attracted much attention. ${ }^{4-48}$ Colonization of the introduced TCC-degrading bacteria in the contaminated environments, such as in anoxic sediments, is an important prerequisite for

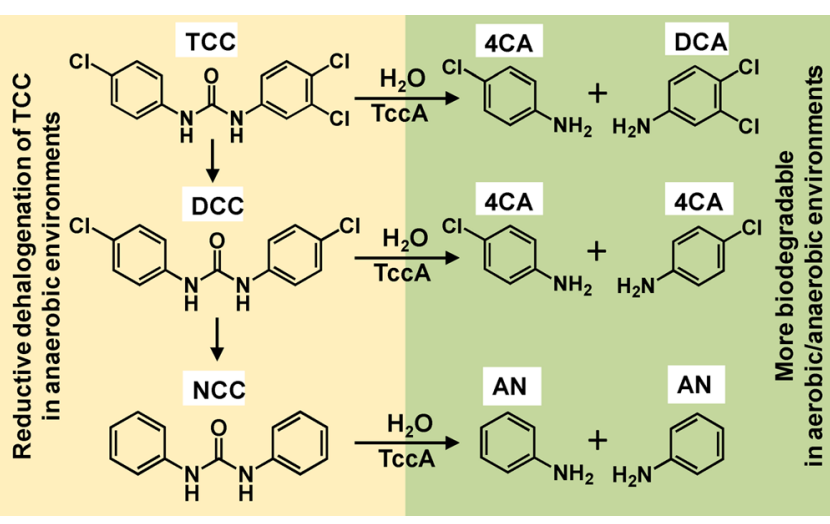

Figure 6. Proposed pathway for the hydrolysis of the two amide bonds of TCC, DCC, and NCC by TccA. The reductive dehalogenation pathway of TCC to generate DCC and NCC that occurs extensively in anaerobic environments is also shown.

efficient bioremediation. Nitrate is generally a thermodynamically favorable electron acceptor for anaerobic respiration. ${ }^{31,49}$ In this study, we demonstrated that strain TCC- 2 was able to 
grow under the anaerobic denitrifying condition accompanied by hydrolysis of TCC and its dehalogenated congeners to the corresponding (chloro)anilines. The hydrolysis of the two amide bonds of TCC and its dehalogenated congeners would primarily facilitate the aerobic/anaerobic mineralization of the more easily biodegradable (chloro)aniline products by indigenous microbes (Figure 6). After all, the biodegradation and mineralization of (chloro)anilines has been studied in a variety of bacteria. ${ }^{50}$ This provides possibilities for the complete bioremediation of TCC in anoxic environments by combining biostimulation and bioaugmentation strategies. Moreover, nitrate-based biostimulation has been shown to accelerate the biodegradation of other organic compounds in contaminated sediments. $^{31,49,51}$ Collectively, the discovery of a novel TCChydrolyzing amidase TccA deepens our understanding of the function of members of the amidase signature enzyme family and provides a potential candidate for the bioremediation of TCC-contaminated environments.

\section{ASSOCIATED CONTENT}

\section{S Supporting Information}

The Supporting Information is available free of charge on the ACS Publications website at DOI: 10.1021/acs.est.6b04885.

Figures S1-S9 and Tables S1-S5. Figures showing the transmission electron micrograph and 16S rRNA genes based phylogenetic tree of strain TCC-2, biotransformation of DCC and NCC under anaerobic conditions, alignment of the amino acid sequence of TccA with other biochemically characterized and putative amidases, HPLC spectrogram of 4CA and DCA accumulation from TCC hydrolysis with E. coli BL21 harboring pET29a-tccA, the transformation efficiency of TCC to 4CA and DCA with the purified TccA, SDS-PAGE, and MALDI-TOF/MS analysis of the purified TccA, UPLC-MS analysis of diflubenzuron, chlorpropham, and 4-bromophenylurea and forchlorfenuron hydrolysis products by the purified TccA. Tables showing the concentrations of TCC detected in various aquatic environments, sludge, and sediments, TCC biotransformation and 4CA and DCA formation rate constants; the influence of metal ions, chemical detergents, and inhibitors on TccA activity and the substrate spectrum and specificity of TccA (PDF)

\section{AUTHOR INFORMATION}

\section{Corresponding Authors}

*Phone/fax: +86 10 62915515; e-mail: binliang@rcees.ac.cn.

*E-mail: ajwang@rcees.ac.cn.

ORCID

Bin Liang: 0000-0003-1235-3498

Notes

The authors declare no competing financial interest.

\section{ACKNOWLEDGMENTS}

This work was supported by the National Science Foundation for Distinguished Young Scholars of China (No. 51225802), the National Natural Science Foundation of China (No. 31500084), the State Key Laboratory of Urban Water Resource and Environment of Harbin Institute of Technology (No. 2016DX03), the Hundred Talents Program of the Chinese Academy of Sciences (No. 29BR2013001) and the Key Deployment Project of Chinese Academy of Sciences (No. ZDRW-ZS-2016-5-5).

\section{REFERENCES}

(1) Halden, R. U.; Paull, D. H. Co-occurrence of triclocarban and triclosan in U.S. water resources. Environ. Sci. Technol. 2005, 39 (6), $1420-1426$.

(2) McDonnell, G.; Russell, A. D. Antiseptics and disinfectants: activity, action, and resistance. Clin. Microbiol. Rev. 1999, 12 (1), 147179.

(3) Perencevich, E. N.; Wong, M. T.; Harris, A. D. National and regional assessment of the antibacterial soap market: a step toward determining the impact of prevalent antibacterial soaps. Am. J. Infect. Control 2001, 29 (5), 281-283.

(4) Carey, D. E.; Zitomer, D. H.; Hristova, K. R.; Kappell, A. D.; McNamara, P. J. Triclocarban influences antibiotic resistance and alters anaerobic digester microbial community structure. Environ. Sci. Technol. 2016, 50 (1), 126-134.

(5) Heidler, J.; Sapkota, A.; Halden, R. U. Partitioning, persistence, and accumulation in digested sludge of the topical antiseptic triclocarban during wastewater treatment. Environ. Sci. Technol. 2006, 40 (11), 3634-3639.

(6) Sun, Q.; Li, M.; Ma, C.; Chen, X.; Xie, X.; Yu, C. P. Seasonal and spatial variations of PPCP occurrence, removal and mass loading in three wastewater treatment plants located in different urbanization areas in Xiamen, China. Environ. Pollut. 2016, 208, 371-381.

(7) Souchier, M.; Benali-Raclot, D.; Benanou, D.; Boireau, V.; Gomez, E.; Casellas, C.; Chiron, S. Screening triclocarban and its transformation products in river sediment using liquid chromatography and high resolution mass spectrometry. Sci. Total Environ. 2015, 502, 199-205.

(8) Pycke, B. F.; Roll, I. B.; Brownawell, B. J.; Kinney, C. A.; Furlong, E. T.; Kolpin, D. W.; Halden, R. U. Transformation products and human metabolites of triclocarban and triclosan in sewage sludge across the United States. Environ. Sci. Technol. 2014, 48 (14), 78817890.

(9) Ahn, K. C.; Kasagami, T.; Tsai, H. J.; Schebb, N. H.; Ogunyoku, T.; Gee, S. J.; Young, T. M.; Hammock, B. D. An immunoassay to evaluate human/environmental exposure to the antimicrobial triclocarban. Environ. Sci. Technol. 2012, 46 (1), 374-381.

(10) Lv, M.; Sun, Q.; Xu, H.; Lin, L.; Chen, M.; Yu, C. P. Occurrence and fate of triclosan and triclocarban in a subtropical river and its estuary. Mar. Pollut. Bull. 2014, 88 (1-2), 383-388.

(11) Halden, R. U. On the need and speed of regulating triclosan and triclocarban in the United States. Environ. Sci. Technol. 2014, 48 (7), 3603-3611.

(12) Zhu, S.; Chen, H. The fate and risk of selected pharmaceutical and personal care products in wastewater treatment plants and a pilotscale multistage constructed wetland system. Environ. Sci. Pollut. Res. 2014, 21 (2), 1466-1479.

(13) Targeted national sewage sludge survey statistical analysis report, EPA-822-R-08-018; U.S. EPA: Washington, D.C., 2009.

(14) Souchier, M.; Casellas, C.; Ingrand, V.; Chiron, S. Insights into reductive dechlorination of triclocarban in river sediments: Field measurements and in vitro mechanism investigations. Chemosphere 2016, 144, 425-432.

(15) Venkatesan, A. K.; Pycke, B. F.; Barber, L. B.; Lee, K. E.; Halden, R. U. Occurrence of triclosan, triclocarban, and its lesser chlorinated congeners in Minnesota freshwater sediments collected near wastewater treatment plants. J. Hazard. Mater. 2012, 229-230, $29-35$.

(16) Miller, T. R.; Heidler, J.; Chillrud, S. N.; DeLaquil, A.; Ritchie, J. C.; Mihalic, J. N.; Bopp, R.; Halden, R. U. Fate of triclosan and evidence for reductive dechlorination of triclocarban in estuarine sediments. Environ. Sci. Technol. 2008, 42 (12), 4570-4576.

(17) Ying, G. G.; Yu, X. Y.; Kookana, R. S. Biological degradation of triclocarban and triclosan in a soil under aerobic and anaerobic conditions and comparison with environmental fate modelling. Environ. Pollut. 2007, 150 (3), 300-305.

(18) Snyder, E. H.; O'Connor, G. A.; McAvoy, D. C. Toxicity and bioaccumulation of biosolids-borne triclocarban (TCC) in terrestrial organisms. Chemosphere 2011, 82 (3), 460-467. 
(19) Gledhill, W. Biodegradation of 3, 4, 4'-trichlorocarbanilide, TCC®, in sewage and activated sludge. Water Res. 1975, 9 (7), 649654.

(20) Miller, T. R.; Colquhoun, D. R.; Halden, R. U. Identification of wastewater bacteria involved in the degradation of triclocarban and its non-chlorinated congener. J. Hazard. Mater. 2010, 183 (1-3), 766772.

(21) Mulla, S. I.; Hu, A.; Wang, Y.; Sun, Q.; Huang, S. L.; Wang, H.; $\mathrm{Yu}$, C. P. Degradation of triclocarban by a triclosan-degrading Sphingomonas sp. strain YL-JM2C. Chemosphere 2016, 144, 292-296.

(22) Liang, B.; Li, R.; Jiang, D.; Sun, J.; Qiu, J.; Zhao, Y.; Li, S.; Jiang, J. Hydrolytic dechlorination of chlorothalonil by Ochrobactrum sp. CTN-11 isolated from a chlorothalonil-contaminated soil. Curr. Microbiol. 2010, 61 (3), 226-233.

(23) Wang, A. J.; Cheng, H. Y.; Liang, B.; Ren, N. Q.; Cui, D.; Lin, N.; Kim, B. H.; Rabaey, K. Efficient reduction of nitrobenzene to aniline with a biocatalyzed cathode. Environ. Sci. Technol. 2011, 45 (23), 10186-10193.

(24) Zhang, J.; Yin, J. G.; Hang, B. J.; Cai, S.; He, J.; Zhou, S. G.; Li, S. P. Cloning of a novel arylamidase gene from Paracoccus sp. strain FLN-7 that hydrolyzes amide pesticides. Appl. Environ. Microbiol. 2012, 78 (14), 4848-4855.

(25) Wang, G.; Li, R.; Li, S.; Jiang, J. A novel hydrolytic dehalogenase for the chlorinated aromatic compound chlorothalonil. J. Bacteriol. 2010, 192 (11), 2737-2745.

(26) Purkhold, U.; Pommerening-Roser, A.; Juretschko, S.; Schmid, M. C.; Koops, H. P.; Wagner, M. Phylogeny of all recognized species of ammonia oxidizers based on comparative 16S rRNA and amoA sequence analysis: implications for molecular diversity surveys. Appl. Environ. Microbiol. 2000, 66 (12), 5368-5382.

(27) Tamura, K.; Peterson, D.; Peterson, N.; Stecher, G.; Nei, M.; Kumar, S. MEGA5: molecular evolutionary genetics analysis using maximum likelihood, evolutionary distance, and maximum parsimony methods. Mol. Biol. Evol. 2011, 28 (10), 2731-2739.

(28) Letunic, I.; Bork, P. Interactive tree of life (iTOL) v3: an online tool for the display and annotation of phylogenetic and other trees. Nucleic Acids Res. 2016, 44 (W1), 242-245.

(29) Qiu, J.; Ma, Y.; Wen, Y.; Chen, L.; Wu, L.; Liu, W. Functional identification of two novel genes from Pseudomonas sp. strain HZN6 involved in the catabolism of nicotine. Appl. Environ. Microbiol. 2012, 78 (7), 2154-2160.

(30) Schmittgen, T. D.; Livak, K. J. Analyzing real-time PCR data by the comparative $C^{\mathrm{T}}$ method. Nat. Protoc. 2008, 3 (6), 1101-1108.

(31) Xu, M.; He, Z.; Zhang, Q.; Liu, J.; Guo, J.; Sun, G.; Zhou, J. Responses of aromatic-degrading microbial communities to elevated nitrate in sediments. Environ. Sci. Technol. 2015, 49 (20), 1242212431.

(32) Lee, S.; Park, E. H.; Ko, H. J.; Bang, W. G.; Kim, H. Y.; Kim, K. H.; Choi, I. G. Crystal structure analysis of a bacterial aryl acylamidase belonging to the amidase signature enzyme family. Biochem. Biophys. Res. Commun. 2015, 467 (2), 268-274.

(33) T'Syen, J.; Tassoni, R.; Hansen, L.; Sorensen, S. J.; Leroy, B.; Sekhar, A.; Wattiez, R.; De Mot, R.; Springael, D. Identification of the amidase $\mathrm{BbdA}$ that initiates biodegradation of the groundwater micropollutant 2, 6-dichlorobenzamide (BAM) in Aminobacter sp. MSH1. Environ. Sci. Technol. 2015, 49 (19), 11703-11713.

(34) Verlicchi, P.; Zambello, E. Pharmaceuticals and personal care products in untreated and treated sewage sludge: Occurrence and environmental risk in the case of application on soil - A critical review. Sci. Total Environ. 2015, 538, 750-767.

(35) Yu, H.; Tang, H.; Zhu, X.; Li, Y.; Xu, P. Molecular mechanism of nicotine degradation by a newly isolated strain, Ochrobactrum sp. strain SJY1. Appl. Environ. Microbiol. 2015, 81 (1), 272-281.

(36) Murínová, S.; Dercová, K. Potential use of newly isolated bacterial strain Ochrobactrum anthropi in bioremediation of polychlorinated biphenyls. Water, Air, Soil Pollut. 2014, 225 (6), 1-16.

(37) Zhai, Y.; Li, K.; Song, J.; Shi, Y.; Yan, Y. Molecular cloning, purification and biochemical characterization of a novel pyrethroid- hydrolyzing carboxylesterase gene from Ochrobactrum anthropi YZ-1. J. Hazard. Mater. 2012, 221-222, 206-212.

(38) Liang, B.; Wang, G.; Zhao, Y.; Chen, K.; Li, S.; Jiang, J. Facilitation of bacterial adaptation to chlorothalonil-contaminated sites by horizontal transfer of the chlorothalonil hydrolytic dehalogenase gene. Appl. Environ. Microbiol. 2011, 77 (12), 4268-4272.

(39) Danko, A. S.; Saski, C. A.; Tomkins, J. P.; Freedman, D. L. Involvement of coenzyme $\mathrm{M}$ during aerobic biodegradation of vinyl chloride and ethene by Pseudomonas putida strain AJ and Ochrobactrum sp. strain TD. Appl. Environ. Microbiol. 2006, 72 (5), 3756-3758.

(40) Fukuta, Y.; Koizumi, S.; Komeda, H.; Asano, Y. A new aryl acylamidase from Rhodococcus sp. strain Octl acting on $\omega$-lactams: Its characterization and gene expression in Escherichia coli. Enzyme Microb. Technol. 2010, 46 (3), 237-245.

(41) Shen, W.; Chen, H.; Jia, K.; Ni, J.; Yan, X.; Li, S. Cloning and characterization of a novel amidase from Paracoccus sp. M-1, showing aryl acylamidase and acyl transferase activities. Appl. Microbiol. Biotechnol. 2012, 94 (4), 1007-1018.

(42) Bers, K.; Leroy, B.; Breugelmans, P.; Albers, P.; Lavigne, R.; Sorensen, S. R.; Aamand, J.; De Mot, R.; Wattiez, R.; Springael, D. A novel hydrolase identified by genomic-proteomic analysis of phenylurea herbicide mineralization by Variovorax sp. strain SRS16. Appl. Environ. Microbiol. 2011, 77 (24), 8754-8764.

(43) Sapkota, A.; Heidler, J.; Halden, R. U. Detection of triclocarban and two co-contaminating chlorocarbanilides in US aquatic environments using isotope dilution liquid chromatography tandem mass spectrometry. Environ. Res. 2007, 103 (1), 21-29.

(44) Oinuma, K.; Takuwa, A.; Taniyama, K.; Doi, Y.; Takaya, N. Hydrazidase, a novel amidase signature enzyme that hydrolyzes acylhydrazides. J. Bacteriol. 2015, 197 (6), 1115-1124.

(45) Zhou, N. A.; Gough, H. L. Enhanced biological trace organic contaminant removal: A lab-scale demonstration with bisphenol Adegrading bacteria Sphingobium sp. BiD32. Environ. Sci. Technol. 2016, 50 (15), 8057-8066.

(46) Sekhar, A.; Horemans, B.; Aamand, J.; Sorensen, S. R.; Vanhaecke, L.; Bussche, J. V.; Hofkens, J.; Springael, D. Surface colonization and activity of the 2,6-dichlorobenzamide (BAM) degrading Aminobacter sp. strain MSH1 at macro- and micropollutant BAM concentrations. Environ. Sci. Technol. 2016, 50 (18), 1012310133.

(47) Michalsen, M. M.; King, A. S.; Rule, R. A.; Fuller, M. E.; Hatzinger, P. B.; Condee, C. W.; Crocker, F. H.; Indest, K. J.; Jung, C. M.; Istok, J. D. Evaluation of biostimulation and bioaugmentation to stimulate hexahydro-1,3,5-trinitro-1,3,5,-triazine degradation in an aerobic groundwater aquifer. Environ. Sci. Technol. 2016, 50 (14), $7625-7632$.

(48) Helbling, D. E. Bioremediation of pesticide-contaminated water resources: the challenge of low concentrations. Curr. Opin. Biotechnol. 2015, 33, 142-148.

(49) Xu, M.; Zhang, Q.; Xia, C.; Zhong, Y.; Sun, G.; Guo, J.; Yuan, T.; Zhou, J.; He, Z. Elevated nitrate enriches microbial functional genes for potential bioremediation of complexly contaminated sediments. ISME J. 2014, 8 (9), 1932-1944.

(50) Arora, P. K. Bacterial degradation of monocyclic aromatic amines. Front. Microbiol. 2015, 6, 820.

(51) Tang, Y. J.; Carpenter, S.; Deming, J.; Krieger-Brockett, B. Controlled release of nitrate and sulfate to enhance anaerobic bioremediation of phenanthrene in marine sediments. Environ. Sci. Technol. 2005, 39 (9), 3368-3373. 